Electrocardlography

$15.9-3$

\title{
DECOMPOSITION OF HEART RATE VARIABILITY BY ADAPTIVE FILTERING FOR ESTIMATION OF CARDIAC VAGAL TONE
}

\author{
K. Han, J.H. Nagel, B.E. Hurwitz and N. Schneiderman
}

\author{
Behavioral Medicine Research Center, Departments of Biomedical Engineering \& \\ Psychology, University of Miami, Coral Gables, FL 33124
}

Abstract

Heart rate fluctuations resulting from respiration and other influences upon the cardiovascular system are encoded into the patterns of heart rate variability (HRV). The fluctuations due to respiration are called respiratory sinus arrhythmia (RSA). Since RSA is primarily mediated through the autonomic nervous system (ANS), it is of interest to separate RSA from other influences to assess the underlying ANS function. On the other hand, the RSA may obscure heart rate responses to external manipulations in psychophysiological tests. A method of partitioning the HRV signal which can provide quantitative estimate of RSA as well as true heart rate responses without respiratory disturbances for psychophysiological studies is developed. The analysis of HRV signal is performed using an adaptive filtering system. With the simultaneously recorded respiration signal as a reference input, the HRV signal can be separated into two components, RSA and fluctuation due to other influences. After the separation, the variance of RSA, an estimate of cardiac vagal tone (ECVT), is readily obtained. The performance of the system was evaluated using artificial test signals as well as real HRV data. As a time domain approach, the method is simple, fast and robust.

\section{Introduction}

Several researchers have reported that phasic changes in respiration have a gating influence on cardiac vagal efferent activity such that during the expiratory phase of respiration a slowing of heart rate (HR) is observed [1]. The oscillatory influence of respiration on HR has been referred to as respiratory sinus arrythmia (RSA). The magnitude of the HR variability (HRV) within the spectral range of respiration has been used as a measure of the strength of parasympathetic activity of the autonomic nervous system [3]. In addition, RSA has also been employed to index central nervous system cholinergic functioning reflecting cognitive processes such as attention.

Spectral analysis has been used to partition the HR variation occurring at the respiratory frequencies [2]. The spectra of respiration and heart rate show high similarities, although the HR spectrum contains prominent frequency bands which are not related to respiration [4]. These findings suggest that in addition to $\mathrm{HRV}$ reflecting vagal cardiac influences, sympathetic and other non-neural factors may contribute to the production of HRV $[2,4]$. Spectral analysis enables the quantitative description of RSA over a specified period. This procedure, however, limits the assessment to more prolonged periods of stable physiological conditions requiring stationarity of means and variances of the signals collected. This limitation prohibits a beat-by-beat assessment of vagal activity, which is desirable in studies examining dynamic cardiorespiratory responsiveness to challenge conditions. Therefore, in the present study we have attempted to develop a methodology to: 1) quantitate the magnitude of respiratory (RSA) and non-respiratory (NRSA) influences on HRV; 2) provide RSA and NRSA measures which are insensitive to violations of signal stationarity, and 3) provide the capability for deriving a continuous beat-by-beat assessment. These goals have been accomplished by developing an adaptive filter for the decomposition of HRV in the time domain.

\section{Adaptive Flitering}

Adaptive filtering is a technique originally developed for estimating signals corrupted by additive noise or interferences [6]. The method uses a primary input containing the desired signal superimposed by additive noise, and a reference input containing the noise which is correlated in some unknown way with the noise in the primary input. The reference input is adaptively filtered and subtracted from the primary input to obtain the best estimated signal. Fig. 1 shows the block diagram of an adaptive

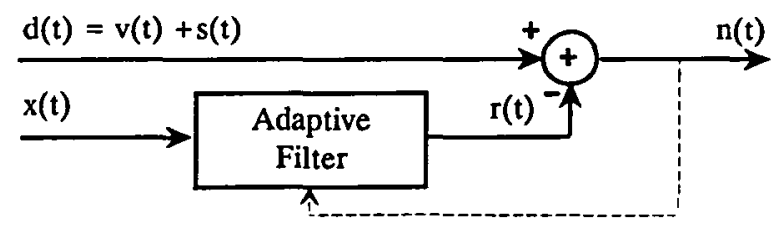

Figure 1 Block diagram of adaptive filtering system.

filtering system. The primary input $d(t)$ is considered to consist of two uncorrelated components $v(t)$ and $s(t)$, and $v(t)$ is correlated with the reference input $x(t)$ while $s(t)$ is not. The reference input $x(t)$ is processed by an adaptive filter with adjustable coefficients and its output is $r(t)$. The system output $n(t)$ is obtained by subtraction of $r(t)$ from $d(t)$. By feeding the output signal $n(t)$ back to the adaptive filter and adjusting its coefficients, the mean square error of the system $E(n(t)-s(t))^{2}=E(v(t)$ $r(t))^{2}$ can be minimized. Therefore, $n(t)$ is the best estimate in the least square sense to $s(t)$, and at the same time, the $r(t)$ is the best estimate of $v(t)$. Adaptive filtering before subtracting allows the treatment of input signals that are deterministic or stochastic, stationary or time variable. According to the described model, the HRV signal has two distinct and uncorrelated components, RSA, which is correlated with respiration, and the fluctuations due to other influences. Therefore, adaptive filtering can be readily applied to analyze HRV signals. Taking the HRV signal as primary input $d(t)$, with $v(t)$ representing RSA and $s(t)$ representing other variations, and the simultaneously recorded respiratory signal as reference input $x(t)$, the two component signals $v(t)$ and $s(t)$ will be best estimated by the adaptive filtering system outputs $r(t)$ and $n(t)$, respectively. As a result, the HRV signal is partitioned into $r(t)$, an estimate of RSA, the component due to respiration, and $n(t)$, an estimate of fluctuations due to other influences.

\section{Implementation of An Adaptive Filtering System}

The adaptive filtering system for the analysis of HRV signals has been implemented on a digital computer (PS/2) using the LMS algorithm [6]. Since the only measurable information on HRV is contained in the cardiac event series which is defined only at the moment when a characteristic cardiac event (e.g. R-peak) appears, it is necessary for further signal processing to convert this time series into a continuously defined HRV signal. A physiologically plausible morel describing a HRV signal influencing and governing the heart rate is the Integral Pulse Frequency Modulation (IPFM) model [5]. The instantaneous heart rate (IHR), which is obtained by constant interpolation of the intervals between two heart beats, is consistent with this model and regarded as an appropriate signal for the time-domain representation of HRV [5]. A respiration signal is obtained using a mercury strain gauge fastened on the subject's chest. Both HRV and respiration signals are digitized with a sampling rate of $4 \mathrm{~Hz}$

The reference input vector $X_{i}$ and filter coefficient vector $W$ defined 
as

$$
\begin{aligned}
x_{i} & =\left[x_{i}, x_{i-1}, \ldots, x_{i-p}\right]^{T} \\
w & =\left[w_{0,}, w_{1}, \ldots, w_{p}\right]^{T}
\end{aligned}
$$

where $p$ is the order of the filter. The filter output $r_{i}$ and system output $n_{i}$ are

$$
\begin{aligned}
& r_{i}=X^{T}{ }_{i} W=W^{T} X_{i} \\
& z_{1}=d_{i}-r_{i}
\end{aligned}
$$

According to the LMS algorithm, the filter coefficients are updated by $w_{i+1}=w_{i}+2 \mu n_{i} X_{i}$

where $W_{i+1}$ and $W_{i}$ are next and present weight vectors, respectively, $\mu$ is the factor that controls stability and the rate of convergence. It has been shown that with arbitrary initial weights, the algorithm will converge to its mean and remain stable as long as the parameter $\mu$ is

$$
\mu_{\max }>\mu>0
$$

where $\mu_{\max }$ is reciprocal of the maximum eigenvalue of the reference auto-correlation matrix [6]. Since the algorithm is based on the steepest descent method and actually makes use of gradients of mean-square error (MSE) functions in searching the optimal solutions, the average excess MSE, in processing time varying signals, consists of two components due to gradient noise and weight vector lag. With the $\mu$ satisfying the convergence condition, the former is proportional to $\mu$ while the latter is inversely proportional to $\mu$. The larger $\mu$, the faster the convergence and therefore the smaller the tracking error due to lag. However the larger $\mu$ will result in larger gradient noise. The optimal values for parameters $p$ and $\mu$ are problem dependent.

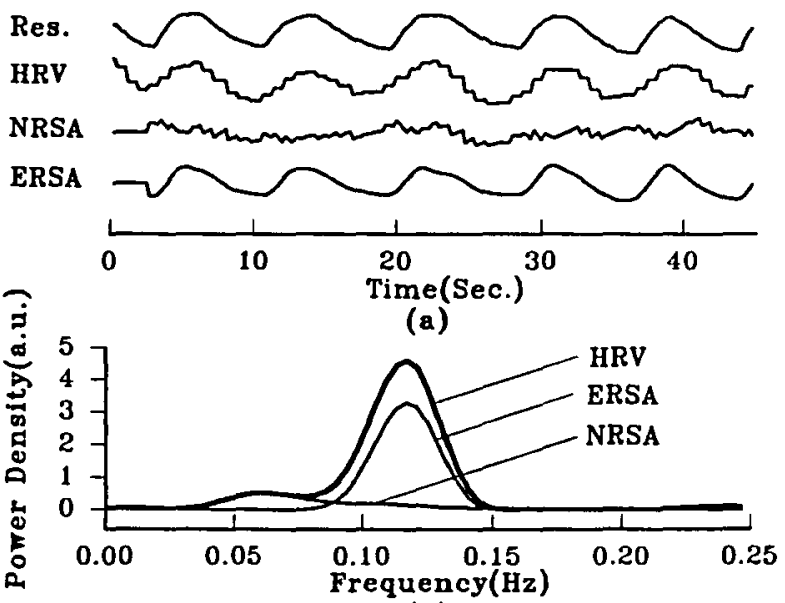

(b)

Figure 2 Decomposition of a $H R V$ signal using the adaptive filtering system. (a)Res.--respiration; HRV-heart rate variability; NRSA-estimated non respiratory fluctuations; $E R S A$-estimated respinatory fluctuations; (b) Power spectra of HRV, NRSA and ERSA.

For the analysis of HRV signals, we use $p=10$ and $\mu=0.005$. Fig. 2(a) shows a decomposition of a real HRV signal by the adaptive filtering system. ERSA represents the estimated HR fluctuations correlated to respirations and NRSA represents the estimated non-respiratory HR fluctuations.

\section{Performance Test}

The performance of the system was tested using test signals which were generated according to a simple HRV model. The NRSA component was simulated using a sinusoid with a frequency of $0.1 \mathrm{~Hz}$ and corrupted by random noise. The respiration signal was simulated by a $0.19 \mathrm{~Hz}$ sinusoid with its amplitude partially modulated by another very low frequency $(0.01 \mathrm{~Hz})$ and a random phase sinusoid which is simulating a time varying process in respiration. The RSA component was obtained by passing the respiration signal through a low-pass filter, and the HRV signal was formed by adding NRSA and RSA components.

Test 1: Precision of waveform estimation
Here the NRSA and RSA components are considered as waveforms to be estimated using the adaptive filtering system. After the estimated waveforms were obtained, they were compared with the known components and the normalized least square errors (LSE) were calculated. The average LSE for NRSA and RSA component estimations obtained from 10 sets of test signals were $3.4 \%$ and $3 \%$, respectively.

Test 2: Precision of variance estimations

In this test, the determination of variances of signals was analyzed. After the estimated signals were obtained, their variances were calculated and compared with corresponding variances of the true components. The average relative errors in estimating variances of NRSA and RSA components for the same sets of test signals were $2.1 \%$ and $4.1 \%$, respectively.

Estimates of Cardiac Vagal Tone

Since RSA is primarily mediated through the vagus, the measurement of RSA can be used as an index of cardiac vagal tone [1]. One measure of RSA, which was originally obtained in the frequency domain using the socalled V-hat procedure, is the variation of the HRV signal falling into the spectral band of respiration [3]. By decomposing HRV using the adaptive filter, the signal describing the fluctuations due to respiration can be obtained. The variance of this signal represents the estimate of cardiac vagal tone (ECVT), and is readily calculated. As a time domain approach, it is simple, fast and robust. Compared to the V-hat procedure, the advantages are: (1) capability of continuously tracking time varying signals in HRV analysis while these signals are treated as stationary in the spectral analysis approach. The precision of estimation is improved. Following the V-hat procedure (spectra calculated via Fast Fourier Transform(FFT) and Hamming window) to estimate variances of RSA components of test signals, even in cases where the RSA and NRSA components are well separated in the spectra, the average relative error obtained was about $20 \%$. The error for the same measurement using the time domain approach was reduced to $4.1 \%$. (2) capability of separating uncorrelated components with overlapping spectra. Since the decomposition using adaptive filtering is performed based on the correlations with respiration, overlap of the two component spectra are allowed. Fig. 2(b) shows spectra of a real HRV signal and its two component signals obtained using adaptive filtering. The spectra of the component signals partially overlap. In contrast to the V-hat procedure, the adaptive filter does not require arbitrary decisions of bandwidth boundaries in the HRV spectrum thus eliminating this factor as a source of error in the estimation of cardiac vagal tone.

\section{References}

[1] P.G. Katona, J.W. Poitras, G.O. Barnett \& B.S. Terry, Cardiac Vagal Efferent Activity and Heart Period in the Carotid Sinus reflex, Am J Physiol, 218, 1030-1037, 1970

[2] G.F. Chess, R.M.K. Tam \& F.R. Calaresu, Influence of Cardiac Neural Inputs on Rhythmic Variations of Heart Period in the Cat, Am J Physiol, 228, 775-780, 1975

[3] P.M. McCabe, B.G. Yongue, S.W. Porges \& P.K. Ackles, Changes in Heart Period, Heart Period Variability, and a Spectral analysis estimate of respiratory Sinus Arrhythmias during Aortic Nerve Stimulation in Rabbits, Psychophysiology, 21, 149-158, 1984

[4] S. Akselrod, D. Gordon and et al, Power Spectrum Analysis of Heart Rate Fluctuation: A Quantitative Probe of Bcat-to-Beat Cardiovascular Control, Science, 213, 2200-222, 1981

[5] R.W. De Boer, J.M. Karemaker, \& J. Dtrackee, Description of Heart-Rate Variability Data in Accordance With a Physiological Model for the Genesis of Heartbeats, Psychophysiology, 22, 147-155, 1985

[6] B. Widrow, J.R. Glover Jr, J.M. McCool, and et al, Adaptive Noise Cancelling Principles and Applications, Proc IEEE, 63, 1692-1716, 1975

Kedu Han

Behavioral Medicine, University of Miami

P.O. Box 248185, Coral Gables, FL 33124 